\title{
Pregnancy experience and mental state of women undergoing fetoscopic surgery due to Twin-to-twin transfusion syndrome:A mixed methods study in Chinese women
}

\author{
Lijie Ren ${ }^{1}$, Xianghong Sun ${ }^{2}$, Yabin Zhang ${ }^{3}$, Chunli Huang ${ }^{4}$, Yan Yang ${ }^{2}$, and Shaowei Yin ${ }^{1}$ \\ ${ }^{1}$ Affiliation not available \\ ${ }^{2}$ The Affiliated Shengjing Hospital of China Medical University \\ ${ }^{3}$ School of Nursing, Lanzhou university \\ ${ }^{4}$ Fuwai Central China Cardiovascular Hospital
}

September 28, 2020

\begin{abstract}
Objective To explore the pregnancy experience and mental state of women undergoing fetal surgery due to twin-to-twin transfusion syndrome. Design A mixed methods study collecting: both quantitative and qualitative data Setting The Affiliated Shengjing Hospital of China Medical University Population Women undergoing fetoscopic surgery due to Twin-to-twin transfusion syndrome Main Outcome Measures Quantitative data was collected using the Impact of Event Scale-Revised questionnaire, the Self-Rating Anxiety Scale, and the Pregnancy Stress Scale. Qualitative data was collected using a semi-structured interview Results The emotional reaction of women undergoing fetoscopic surgery due to twin-to-twin transfusion syndrome was long-term, and the adverse emotions did not diminished over time. After surgery, patients were constantly worried about the health of the fetuses, and the lack of disease information will aggravate their uncertainty and worry. Twin transfusion syndrome may also cause a post-traumatic stress reaction in women, but for protecting the surviving fetuses, they utilized positive ways to regulate negative emotions, so postoperative worry about fetuses did not cause them much anxiety. Conclusion Gestational emotional reaction of women undergoing TTTS fetoscopic surgery is complicated. According to our research, optimizing the referral system and strengthening disease information support could be a good way for clinical staff to improve these women's mental state during pregnancy and avoid emotion to fluctuate violently,especially for people with weaker fertility.
\end{abstract}

Pregnancy experience and mental state of women undergoing fetoscopic surgery due to Twin-to-twin transfusion syndrome:A mixed methods study in Chinese women

Authors

Ren Lijie $^{\mathrm{a}}$.E-mail:renlijie0623@163.com

Sun Xianghong ${ }^{\mathrm{a}}$.E-mail:750019113@qq.com

Zhang Yabin ${ }^{\mathrm{b}}$.E-mail:zyb4322@163.com.

Huang Chunlic. E-mail:1341826260@qq.com

Yang Yan ${ }^{\mathrm{a}}$.E-mail:yangyan1776@163.com

Yin Shaowei ${ }^{\mathrm{a}}$.E-mail:yinshaowei8403@163.com

a: The Affiliated Shengjing Hospital of China Medical University. 36 Shanhao Road,Heping District,Shenyang City,Liaoning Province,China.CO 110004 
b: School of Nursing, Lanzhou university. Address:No. 28, Yanxi road, Lanzhou City, Gansu Province , China. CO 730030

c:Fuwai Central China Cardiovascular Hospital. FuWai Road,ZhengZhou City,HeNan Province,China.CO 45000

Corresponding authors:

Yang Yan: The Affiliated Shengjing Hospital of China Medical University. 36 Shanhao Road,Heping District,Shenyang City,Liaoning Province,China.CO 110004

E-mail:yangyan1776@163.com cell-phone number:18940251776

Yin Shaowei :The Affiliated Shengjing Hospital of China Medical University. 36 Shanhao Road,Heping District,Shenyang City,Liaoning Province,China.CO 110004

E-mail: yinshaowei8403@163.com cell-phone number:18940258403

Running Title: A Mixed Methods study about TTTS

Pregnancy experience and mental state of women undergoing fetoscopic surgery due to Twin-to-twin transfusion syndrome:A mixed methods study in Chinese women

Objective To explore the pregnancy experience and mental state of women undergoing fetal surgery due to twin-to-twin transfusion syndrome.

Design A mixed methods study collecting: both quantitative and qualitative data

Setting The Affiliated Shengjing Hospital of China Medical University

Population Women undergoing fetoscopic surgery due to Twin-to-twin transfusion syndrome

Main Outcome Measures Quantitative data was collected using the Impact of Event Scale-Revised questionnaire, the Self-Rating Anxiety Scale, and the Pregnancy Stress Scale. Qualitative data was collected using a semi-structured interview

Results The emotional reaction of women undergoing fetoscopic surgery due to twin-to-twin transfusion syndrome was long-term, and the adverse emotions did not diminished over time. After surgery, patients were constantly worried about the health of the fetuses,and the lack of disease information will aggravate their uncertainty and worry. Twin transfusion syndrome may also cause a post-traumatic stress reaction in women, but for protecting the surviving fetuses ,they utilized positive ways to regulate negative emotions, so postoperative worry about fetuses did not cause them much anxiety.

Conclusion Gestational emotional reaction of women undergoing TTTS fetoscopic surgery is complicated. According to our research, optimizing the referral system and strengthening disease information support could be a good way for clinical staff to improve these women's mental state during pregnancy and avoid emotion to fluctuate violently,especially for people with weaker fertility.

Funding: This work was supported by the National Key Research \&Department Program of China,No.2018YFC1002902

Keywords Twin-to-twin transfusion syndrome; Mixed Methods research;fetoscopic surgery; pregnancy experience; psychological state

\section{Introduction}

Twin-to-twin transfusion syndrome (TTTS) is a serious complication of monochorionic diamniotic

(MCDA) pregnancy, which can cause serious damage to multiple fetal systems . ${ }^{1,2}$ Fetoscopic laser coagulation(FLC) has become the standard treatment for TTTS $;^{3}$ however, the survival rate of both twins after treatment is not ideal and the incidence of preterm birth is very high ${ }^{4}$ Based on the characteristics of the 
disease and the current situation of treatment approach, in addition to surgical stress, women with TTTS may experience other adverse events such as intrauterine death of one or both two fetuses, extremely premature birth, and poor prognosis of fetuses during pregnancy. Their emotional reactions during pregnancy are more complex and fluctuation.$^{5}$

Previous researches has demonstrated that that negative emotional state during pregnancy is a risk factor for adverse pregnancy outcomes $;{ }^{6}$ therefore, as the treatment technology of each fetal medical center is becoming mature, we should also can try to improve the pregnancy outcomes by improving the gestational mental state of the mother. However, we know little about the pregnancy experience of women undergoing TTTS and accepting fetoscopic surgery. Current researches indicate that these women may have anxiety and sadness during pregnancy, though, we know little about the extent of these adverse emotions and specific feelings and needs during pregnancy $.5,7,8$ For women with such a complicated pregnancy experience, we must understand their pregnancy experience in detail before we can possibly provide them with targeted psychological support.

Mixed method research refers to the simultaneous existence of quantitative and qualitative research in one research, through data integration to realize the complementary advantages of qualitative research and quantitative research, and to achieve a comprehensive and in-depth explanation of a complex problem $.9,{ }^{10}$ This study utilized a Mixed method to examine the psychological state and pregnancy experience of women undergoing fetoscopic surgery due to Twin-to-twin transfusion syndrome. Semi-structured interview was used to explore their detailed pregnancy experience and difficulties caused by the disease; quantitative research was used to explore their emotional state and degree. The aim of this research is to help clinical staff to develop psychological support strategies and optimize referral procedures for women undergoing TTTS.

\section{Methods}

\section{The general design}

This study is a mixed method research. For the quantitative data collection, a questionnaire was used to test the psychological state of participants at around postoperative week 1, 5, and 9, and again at week 5 after delivery. For qualitative data collection, we utilized semi-structured interview method, and the interview time was selected around the 31 gestational week(this time was chosen to be close to the delivery date as possible)or after delivery. In the analysis stage, we combined quantitative data and qualitative data, so that the two data types could support and explain one another..

\section{Setting and sampling method}

This research was conducted in Shengjing Hospital Affiliated to China Medical University between February 2019 and July 2020. To maximize the sample size, the quantitative study included all eligible women during research while. Purpose sampling was used for the qualitative data, women who were willing to share their pregnancy experience and provide more information were selected. Interviewees must had heterogeneity,such as included women with different conception methods - different pregnancy experiences and different regions.

\section{Participants and eligibility}

Participants were eligible for the quantitative aspect of this study if they met the criteria 1)they were diagnosed as TTTS and accepting FLC; 2 ) they were $>18$ years of age and able to complete the questionnaire independently. The qualitative aspect of this study selected women who were willing to participate in the interrviews and complete all aspects of the all recording basing on the participants of quantitative research. However, women with existing psychological problems were not selected.

\section{Data collection}

\section{Quantitative data}

The quantitative data collection questionnaires included: 1) basic information including gestational age, maternal age, education level, income, number of existing offspring, conception method, and history of 
previous pregnancy; 2) Impact of Event Scale-Revised (IES-R); 3) Self-Rating Anxiety Scale (SAS); and 4) Pregnancy Stress Scale. The IES-R is used to evaluate the severity of post-traumatic stress and higher score represent a greater impact of certain life events. ${ }^{11}$ For the IES-R, a score over 19 is the standard of high clinical attention and a patient with a score over 35 is more likely to be diagnosed with post-traumatic stress disorder (PTSD) ; ${ }^{12}$ The SAS is used to assess the subjective feelings of adults in the past week ${ }^{13}$ The Pregnancy Stress Scale was compiled by Chinese scholar Chen Zhanghui and is used to evaluate a women's stress during pregnancy. ${ }^{14}$ This scale contains 30 items which mainly measure the stress caused by identification of parents' role, the stress caused by ensuring the health and safety of mother and fetus, and the stress caused by body's changes. Items in the Pregnancy Stress Scale are judged on a four point system:0 $=$ no pressure, $1=$ mild pressure, $2=$ moderate pressure, and $3=$ severe pressure. Total scores of all items are directly proportional to pressure level. See Appendix S 1 for the complete questionnaire.

\section{Qualitative data collection}

Qualitative data were collected via a semi-structured interview method. To ensure the quality of data and the depth of the interview, we established a trusting relationship with the patient and practiced interview skills prior to the interview.${ }^{15}$ The interview guide was developed according to the purpose of the research, to previously published research, and to a preliminary conversation with women experiencing TTTS.$^{16}$ Main questions included: Could you talk about how TTTS was diagnosed (disease information/feelings)? What was your experience during the whole treatment process (including the referral process and treatment decisions)? What was your experience during postoperative pregnancy (including feelings during reexamination)? During the interview, we asked the interviewees to draw a picture reflecting their emotional state during pregnancy. The best emotion is represented by 10 points, the worst emotion is represented by -10 points, and the scoring interval is not limited. After the interview, we immediately recorded our interview experience and the interviewee's overall emotional state. Data collection and analysis were carried out at the same time, and we stopped the interview when new themes stopped emerging.

\section{Data analysis}

\section{Quantitative data analysis}

Quantitative data was analysed with SPSS 20.0software.Descriptive analysis of measurement data was expressed as mean \pm standard deviation, and enumeration data was expressed by frequency and percentages.The trend of scale score in different period was investigated by repeated measurement data analysis of variance; anxiety scores of participants and normal anxiety norms of Chinese pregnant women were compared by one-sample t-test .

\section{Qualitative data analysis}

Audio material was transcribed within 24 hours of the interview and recorded the overall emotional state and tone changes of each interviewee. Data was analysed according to the Colaizzi method. ${ }^{17}$ All transcribed data was coded independently by two researchers, and different codes were dealt with after negotiation. In the analysis process, we recorded reading notes and systematically explained the process of forming the themes, and all these recordings were sent to a third researcher for examining the rationality. Finally, we returned these themes and the explanation of these themes to some participants to ensure that each theme met the true meaning of the interviewees ${ }^{18}$ 。

\section{Result}

\section{Demographic characteristics}

A total of 19 women participated in this research, and nine of them received semi-structured interviews. The research flow chart is shown in Figure 1. Nearly $50 \%$ of participants had a history of adverse pregnancy; $90 \%$ did not have existing offspring at the time of the research; the successful delivery rate of all participants receiving FLP was $78.95 \%$ ( Table 1).

Main results of quantitative research 
Table 2 shows the four measurement results of the IES-R, SAS, the Pregnancy Stress Scale, and the sample size in each test. The score of IES-R suggests that TTTS may result in a post-traumatic stress response. Items in the Pregnancy Stress Scale about the health of the fetuses have higher scores including items such as premature birth, fetal weight, fetal safety, and fetus abnormity. The first postoperative anxiety score of women undergoing FLC was higher than that of typical Chinese pregnant women $(t=4, \mathrm{P}<0.001)$; however, there was no significant difference between the additional two anxiety scores and the norm $(\mathrm{t}=0.312, \mathrm{P}>0.5$; $\mathrm{t}=0.365, \mathrm{P}>0.5$ ); the repeated measurement data analysis of variance on the nine complete data (every woman accepted four tests) found that the post-traumatic stress level $(\mathrm{F}=0.533, \mathrm{P}=0.568)$, anxiety level $(\mathrm{F}=2.738, \mathrm{P}=0.109)$, and pregnancy stress $(\mathrm{F}=0.286, \mathrm{P}=0.622)$ of women undergoing FLC due to TTTS did not change over time.

\section{Qualitative research results}

Theme 1 Emotional reactions during pregnancy: The main characteristics of adverse emotional reactions were long-term and fluctuating, which could be reflected in the emotional state diagram. The emotions of the four interviewees experienced several fluctuations during pregnancy, when the fetus's health status changed significantly, they had to bear great conversion between joy and sadness in a short time. And it was difficult for the postoperative emotions to return to the pre-diagnosis level ( Figure 2).

\section{Mingled joy and worry}

Regardless of the method of conception, the interviewees were shocked and happy when they first learned twin pregnancy.However, for women with adverse pregnancy history or conception through assisted reproduction, apart from being happy, they were more cautious about the pregnancy. They were "especially worried about uterine contractions and miscarriage and asked for leave for the fetal safety after pregnancy." Clinicians may pay more attention to MCDA pregnancy because of its high risk. When fetal indicators were normal, doctors' general notification of the risks of MCDA pregnancy would not cause women much worry about fetal health. However, when doctors informed MCDA pregnancy women the risk of TTTS specifically or they learned about the existence of TTTS through another means, these women may worry about TTTS before the diagnosis of TTTS. For example, an interviewee said "I learned from the application (APP) that I have the risk of TTTS, then I searched relevant information online frequently and I worried about this disease every day."

\section{The shock of diagnosis}

No matter whether they knew about TTTS before, the interviewees showed shock, sadness, fear, and even self-blame at the time of diagnosis. TTTS develops rapidly, most fetuses' "were normal in the preliminary examination," and when they were suddenly diagnosed with TTTS, these women "were at a loss immediately and kept crying." Because of being unfamiliar with this disease, they thought "it was very serious and both fetuses would die." On the other hand, the treatment of TTTS is carried out in fetal medical centers, and for patients in other places, they didn't know if there was a chance for treatment and didn't know the treatment process. They said that "the pressure on the way to the hospital was the greatest, and we were afraid that the child would die on the way." With these emotions, they also had to find information about the treatment hospital by their own ability during the referral process.

\section{The hope of treatment}

The primary appeal of women who chose FLC was to save their fetuses, and they were eager for treatment as soon as possible. The process of waiting for the operation was the most tormenting, they "could not sleep" and "needed to see a psychologist." They could accept if the fetuses died after the operation, but they could not accept it if the "fetuses died while waiting." The improvement of the fetal health after the operation would give patients great hope, especially when the "results were better every time" during the re-examination. The improvement in fetal health increased their confidence to give birth to two fetuses, and after being discharged from the hospital, they started preparing to welcome the two fetuses, "buying a crib" 
and "preparing a room." But we should be aware that the hope that the fetus got better would become a serious blow if the fetal health later deteriorated.

\section{Uncertainty of fetal health status}

Treatment of TTTS is not once and for all. As the fetus cannot be seen intuitively, postoperative women were very sensitive to the fetal health and any subtle changes caused the patients to worry, this behavior was more obvious after discharge. Women mainly expressed uncertainty and concern about fetal death, premature birth, and fetal development. For example, one interviewee said "the child could not be seen or touched, I didn't know if both fetuses would be delivered successfully, and I'm afraid that my children had sequelae because of premature delivery. So I felt worried every day and lay in bed all the time." Uncertainty and worry were also reflected in the Pregnancy Stress Scale. Items about ensuring the health and safety of both the mother and child had the highest average score, such as worry about premature delivery which caused moderate pressure in women in each test (first test: 2.13; second test: 1.9; third test: 1.67). However, due to the high survival rate of premature babies, gestational age 28 and 32 are the two key time points for neonatal survival, so many women said that "after 28 weeks, there was no such worry."

The sorrow of losing fetus

Women after FLC often found fetuses were dead during a certain examination. The hope that the fetuses would get better during the early stage formed a huge gap with the death of the fetuses. Their "emotion suddenly transformed from heaven to hell" and they would "worry the older fetus would be affected". Excessive grief also triggered physiological reactions such as "the nose bleeding immediately," "the tears couldn't be stopped," and the surviving fetus could not offset the sadness of losing one of the twins. For women who lost two fetuses after surgery, they "cannot accept the facts". But due to different pregnancy experiences, these women had completely different attitudes towards subsequent fertility. For women who had weak fertility and successfully conceived after repeated assisted reproduction, they were extremely disappointed after the death of twins and refused to get pregnant again; For women who conceived naturally or after minimal assisted reproduction, they were eager to get pregnant again, but they were worried about abnormalities appearing in their next pregnancy.

\section{Theme 2 Influencing factors of emotional reaction}

\section{Fetal health status}

Fetal health status was the most direct influencing factor of emotions, Their emotion fluctuated with the fetal health.For example, one interviewee said: "my heart was suspended when I reviewed the Color Doppler Ultrasound, but when doctor said the result was good, my mood instantly relaxed,and I walked easily .". From the emotional state diagram, we could also see that the emotional fluctuation of the interviewees was related to the fetal health.

\section{Information processing}

Being unfamiliar with the disease would aggravate the anxiety and fear of TTTS women. These women were eager to learn about the disease, but professionals cannot provide the required information in time, and the internet became their main approach to learning about the disease. However, it was difficult for women without professional guidance to distinguish the authenticity of online information. A reasonable intake of information would allow them to "know the disease and not worry so much." However, unreasonable information stimulation caused additional anxiety and worry. For example, two interviewees learned about the existence of TTTS through the internet early in pregnancy and they worried about the occurrence of TTTS even before diagnosis. After being diagnosed, receiving too much negative information from the internet increased their uncertainty about the disease. For example, interviewees said: "I often searched the disease online, forums said that the disease always had bad outcomes, and I couldn't see hope after reading this information."

Peer support 
Women who were diagnosed with TTTS needed emotional support from women with the same experience, especially from women diagnosed with TTTS at the same time as them; this type of connection was more intuitive and profound. They thought that their case would follow another patients' disease development and they would experience "serious psychological pressure" when other patient's condition fluctuated. Although the influence would be reduced when patient left hospital environment, the mutual influence among patients must be considered when arranging patient rooms.

The desire for both twins to survive

During the long-term of the pregnancy, these patients perceived the existence of two fetuses and established an attachment to both of them. "This was different from a singleton pregnancy from the beginning." Some women observed the appearance of the fetuses through four-dimensional ultrasound and stated "they were exactly the same, when one was born, I would think of the other"; as such, they didn't want to lose either fetus. However, the current survival rate of both twins after FLC treatment is not ideal. Therefore, the contradiction between the high risk of losing a fetus and a women's desire for the survival of both twins always plagued them.

Theme 3: The main ways of regulating adverse emotions

Unfortunate rationalization

The diagnosis of TTTS was a negative event for women, but in order to protect the surviving fetus or to be relieved from the grief of losing a fetus, most women were inclined to rationalize the disease to relieve their negative emotions. They told themselves "the fetus that I lost was in a bad condition, I could be more uncomfortable if the abnormity appeared after his birth."

\section{Avoiding stressors}

Suppressing negative thoughts and avoiding adverse information were common psychological adjustment mechanisms for patients. Whenever they had bad thoughts about the fetuses, they "negated it immediately and changed to another good thought." Finding negative information about miscarriage and placenta implantation on the internet caused them to worry, and they specifically "didn't read messages in the WeChat group" or "didn't search for information online." This coping mechanism may be the reason for low anxiety scores.

\section{Discussion}

\section{Main findings}

In this study, we found that TTTS may cause post-traumatic stress in women,and their uncertainty and worry about fetal health continued throughout whole pregnancy. They experienced a high pregnancy pressure after the operation, and the pressure source mainly resulted from premature birth, fetal weight, and whether the fetuses were normal. Notably, mothers to protect the surviving fetus, tended to adjust their emotional state positively. For example, the SAS scores indicated high level of anxiety only last for a few days after the operation. This positive adjustment method could also be reflected in the pregnancy emotional state diagram.

In the quantitative results, we found that the standard deviation of each scale score was relatively large, which may indicate that the impact of TTTS on women was quite different. However, due to the limited sample size, we could not analyze the relationship between conception method, pregnancy history, age, and emotional state. However, we found that women with weaker fertility were obviously more worried about the fetal health than young, natural conception women. In addition, this study found that women were relatively unfamiliar with TTTS, and it was difficult for them to obtain professional information support. In the process of searching for information through the internet, a lack of good information processing ability would aggravate their worry about fetal health. How to build an information support system is explained in the interpretation section.

Strengths and weaknesses 
The biggest advantage of our study is achieving the integration of quantitative data and qualitative data, so that the results can reflect the psychological state and pregnancy experience of women undergoing fetoscopic surgery due to TTTS more comprehensive and scientifically. Ours is the first study using related scales to measure the specific level of emotional reactions of such women. It is also the first time that a semistructured interview method has been used to reconstruct the pregnancy experience and deeply understand the emotional reactions and characteristics of such women. However, there are some shortcomings in our research. First, the semi-structured interviews were not conducted after delivery. Although the interview time was close to the delivery, there may be a lack of information about their attitudes towards delivery. The main reason why we chose to conduct interviews before delivery is that most women came from other regions and they would not return to our hospital after delivery. Secondly, the sample size of the quantitative study is limited, so it is hard to conduct in-depth data analyses such as linear regression analysis. However, we are willing to share the original data to help other researchers achieve data integration.

\section{Interpretation}

Women who experience fetal abnormalities, premature birth, or miscarriage may face post-traumatic stress, sadness, anxiety, and/or depression for a long time $\cdot{ }^{19},{ }^{20}$ Women with TTTS have the disadvantages of premature births and miscarriage, and their pregnancy experiences are more complicated. A study indicated that the emotional reactions of whole family experiencing TTTS during pregnancy were like a roller coaster. ${ }^{5}$ Our research also found that the emotional reactions of women undergoing FLP surgery due to TTTS were more complicated. In the interviews, they expressed they were extremely concerned about the fetal health, and they worried about premature delivery, intrauterine death, abnormal fetal development or missed the died fetus for a long time after the operation. In addition to qualitative research, this characteristic of long-term could also be reflected in quantitative data. For example, repeated measures data analysis of variance suggested that the patient's post-traumatic stress state, anxiety level, pregnancy pressure didn't not diminished over time. However, unlike previous studies, due to the advantages of FLP treatment, current patients had less difficulty in choosing treatment methods.For them, the process of waiting for treatment is the most tormented.

Another study investigated the anxiety, depression, and post-traumatic stress of women experiencing TTTS. The results showed that, during pregnancy, $80 \%$ of women thought that they had anxiety symptoms, $66 \%$ of women thought that they had depression symptoms, and $2 \%$ of women thought they had post-traumatic stress.$^{7}$ However, these emotions were not objectively measured and only reflect the subjective perception of the participants. Our study found that women who experienced TTTS had a higher proportion of posttraumatic stress during pregnancy. Among the three measurements during pregnancy, $43.75 \%, 66.67 \%$, and $63.63 \%$ of women had scores greater than 19 on the IES-R. However, our study indicated worry about fetal health did not cause their postoperative long-term anxiety symptoms. The reason for the difference between our study and other study may be related to the changes in the population, study time, and treatment status.

Obtaining sufficient information during pregnancy is necessary for pregnant women to take correct actions, but unreasonable information stimulation can also aggravate their anxiety and worry. ${ }^{21}$ Previous studies pointed out pregnant women are more inclined to obtain pregnancy knowledge through the internet. This observation is consistent with the results of our study.${ }^{22,23}$ Our study also found that patients could not get enough information support in a timely manner at the first-visit hospital and fetal medical center, and they mainly obtained disease information through the internet. However,it was difficult for patients to distinguish the authenticity of the information or find the latest information for unfamiliar diseases like TTTS. Unreasonable information caused greater confusion for patients. For example, a woman saw a lot of negative news in the forum and believed that the chance of fetal survival after treatment was slim, and she lay in bed all the time because of the worry about fetal accident after the operation. However, she was unaware that the information she saw had a great lag and, at present, the treatment outcomes had been significantly improved with the accumulation of doctors' experience. Patients also tend to think that information on the internet is correct and they do not like to discuss that information with their doctor.$^{24}$ 
Based on this observations, after a diagnosis of TTTS, doctors should provide authoritative disease information in a timely manner, and should actively discuss network information with patients to understand their confusions and resolve cognitive errors. After a diagnosis of TTTS, the first-visit hospital should provide basic disease knowledge education to patients and introduce them to the treatment procedures of fetal medicine centers so as to reduce the excessive worry for fetuses and the strangeness with the treatment situation in the referral. In addition, for MCDA pregnant women, medical staff should grasp the appropriate scale when notifying potential complications before the diagnosis of TTTS, so as not to increase the unnecessary worry of pregnant women.

Based on the pregnancy experience and mental state of women undergoing FLC due to TTTS, we believe that a long-term psychological support model should be established, especially for women who are more prone to psychological problems, such as those with weak fertility and loss of fetuses. At present, information support can be regarded as the focus of psychological support, and an online service model should be established to meet patients' needs from different regions. Medical staff can inform patients of possible changes of disease during the later stage and help them accept the characteristics of the disease and not pay too much attention to minor abnormalities. In addition, we should help patients to view the treatment effect rationally and let them accept the possibility of one fetus's survival, and give psychological support in a timely manner to prevent excessive mood swings if the disease deteriorates.

\section{Conclusions}

This study utilized a mixed method research design to explore the pregnancy experience and mental state of women undergoing FLP surgery due to TTTS. We found that adverse emotional reactions caused by TTTS continue throughout pregnancy and these emotions did not weaken over time. TTTS may cause a post-traumatic stress response in women and proper psychological support should be provided. After the operation, they had high pregnancy pressure and worried about the fetal health for a long time. However, in order to protect the surviving fetus, women generally adopted a positive emotion regulation mechanism, so worry for fetus did not make them anxious for a long time. In addition, these women lacked disease information from professionals and we believe disease information education could be an effective way for clinicians to improve their pregnancy experience.

\section{Data sharing and data availability}

We are willing to share all the original data of quantitative research, please contact the corresponding author if necessary.

\section{Acknowledgments}

We thank all the team members who participated in the research. We thank all other hospital staff who helped us during the research process. We are particularly grateful to the women who participated in our study,they dedicated their precious time and willing to share their experiences,so our research can complete smoothly.

Declarations of interest: none

\section{Author Statement}

We declare that this manuscript has not been published or presented elsewhere in part or in entirety and is not under consideration by another journal. All study participants provided informed consent. We have read and understood your journal's policies, and we believe that neither the manuscript nor the study violates any of these.

\section{Contribution to Authorship}

Ren Lijie:I declare that I participated in the design , data collection and statistics, and the writing of the paper. 
Sun Xianghong: I declare that I participated in the data collection and statistics, and the writing of the paper.

Zhang Yabin :I declare that I participated in analysis and writing of qualitative research.

Huang Chunli: I declare that I participated in analysis and writing of qualitative research.

Yang Yan: I declare that I participated in the design ,data collection and the writing of the paper.

Yin Shaowei: I declare that I participated in the design, data collection and statistics, and the writing of the paper.

Details of ethics approval

Ethical approval was obtained from the ethics committee of Affiliated Shengjing Hospital of China Medical University (2018PS20K) on 18 January 2018. The study was conducted according to the principles of the Declaration of Helsinki.

Funding

This work was supported by the National Key Research \&Department Program of China,No.2018YFC1002902

\section{References}

1 Simpson LL. Twin-twin transfusion syndrome. American journal of obstetrics and gynecology 2013;208(1):3-18.

2 Bamberg C, Hecher K. Update on twin-to-twin transfusion syndrome.Best Practice 8 S Research Clinical Obstetrics $\mathscr{E}$ Gynaecology2019;58:55-65.

3 Glennon CL, Shemer SA, Palma-Dias R, Umstad MP. The History of Treatment of Twin-to-Twin Transfusion Syndrome. Twin research and human genetics 2016;19(3):168-74.

4 Murgano D, Khalil A, Prefumo F, Mieghem TV, Rizzo G, Heyborne KD, et al. Outcome of twin-totwin transfusion syndrome in monochorionic monoamniotic twin pregnancy: systematic review and metaanalysis. Ultrasound in obstetrics \& gynecology 2020;55(3):310-7.

5 Fischbein R, Meeker J, Saling J, Chyatte M, Nicholas L. Identifying families' shared disease experiences through a qualitative analysis of online twin-to-twin transfusion syndrome stories. Pregnancy Childbirth $2016 ; 16(163)$.

6 Tomfohr-Madsen L, Cameron EE, Dunkel Schetter C, Campbell T, O'Beirne M, Letourneau N, et al. Pregnancy anxiety and preterm birth: The moderating role of sleep. Health psychology 2019;38(11):1025-35.

7 Falletta L, Fischbein R, Bhamidipalli S, Nicholas L. Depression, anxiety, and mental health service experiences of women with a twin-twin transfusion syndrome pregnancy. Arch Womens Ment Health2018;21(1):7583.

8 Vergote S, Lewi L, Gheysen W, De Catte L, Devlieger R, Deprest J. Subsequent fertility, pregnancy, and gynecologic outcomes after fetoscopic laser therapy for twin-twin transfusion syndrome compared with normal monochorionic twin gestations. American journal of obstetrics and gynecology 2018;218(4):441-7.

9 Shorten A, Smith J. Mixed methods research: expanding the evidence base. Evidence-based nursing 2017;20(3):74-5.

10 Pluye P, Hong Q. Combining the power of stories and the power of numbers: mixed methods research and mixed studies reviews. Annu Rev Public Health 2014;35:29-45. 
11 Kragh AR, Folke F, Andelius L, Ries ES, Rasmussen RV, Hansen CM. Evaluation of tools to assess psychological distress: how to measure psychological stress reactions in citizen responders- a systematic review. BMC emergency medicine 2019;19(64).

12 Ursano RJ, Fullerton CS, Kao TC, Bhartiya VR. Longitudinal assessment of posttraumatic stress disorder and depression after exposure to traumatic death. The Journal of nervous and mental disease1995;183(1):3642 .

13 Zung. A rating instrument for anxiety disorders.Psychosomatics 1971;12(6):371-9.

14 Pan YL,Gao LL,Jin XH. A research about the relationship of pregnancy stress between pregnant women and their spouses. Guangdong Medicine 2004;(10):1209-10.

15 McGrath C, Palmgren P, Liljedahl M. Twelve tips for conducting qualitative research interviews. Medical Teacher2019;41(9):1002-6.

16 Moser A, Korstjens I. Series: Practical guidance to qualitative research. Part 3: Sampling, data collection and analysis. European Journal of General Practice 2018;24(1):9-18.

17 Northall T, Chang E, Hatcher D, Nicholls D. The application and tailoring of Colaizzi's phenomenological approach in a hospital setting. Nurse researcher 2020;28(2):20-5.

18 Korstjens I, Moser A. Series: Practical guidance to qualitative research. Part 4: Trustworthiness and publishing. European Journal of General Practice 2018;24(1):120-4.

19 Pitt P, McClaren BJ, Hodgson J. Embodied experiences of prenatal diagnosis of fetal abnormality and pregnancy termination. Reproductive health matters 2016;24(47):168-77.

20 Yaari M, Treyvaud K, Lee KJ, Doyle LW, Anderson PJ. Preterm Birth and Maternal Mental Health: Longitudinal Trajectories and Predictors. Journal of pediatric psychology 2019;44(6):736-47.

21 Javanmardi M, Noroozi M, Mostafavi F, Ashrafi-Rizi H. Challenges to access health information during pregnancy in Iran: a qualitative study from the perspective of pregnant women, midwives and obstetricians. Reproductive health 2019;16(1).

22 Wexler A, Davoudi A, Weissenbacher D, Choi R, O'Connor K, Cummings H, et al. Pregnancy and health in the age of the Internet: A content analysis of online "birth club" forums. PloS one2020;15(4):e0230947.

23 Snyder A, Neufeld HT, Forbes L. A mixed-methods investigation of women's experiences seeking pregnancy-related online nutrition information. BMC pregnancy and childbirth 2020;20(2):739-51.

24 Sayakhot P, Carolan-Olah M. Internet use by pregnant women seeking pregnancy-related information: a systematic review. BMC pregnancy and childbirth 2016;16(1).

\section{Hosted file}

Tables and figures.pdf available at https://authorea.com/users/362584/articles/483672pregnancy-experience-and-mental-state-of-women-undergoing-fetoscopic-surgery-due-totwin-to-twin-transfusion-syndrome-a-mixed-methods-study-in-chinese-women 